\title{
The COSMIC Imaging Beamline at the Advanced Light Source: a new facility for spectro-microscopy of nano-materials
}

David A. Shapiro $^{1^{*}}$, Richard Celestre ${ }^{1}$, Bjoern Enders ${ }^{1,2}$, John Joseph ${ }^{3}$, Harinarayan Krishnan ${ }^{4}$, Matthew A. Marcus ${ }^{1}$, Kasra Nowrouzi ${ }^{1,2}$, Howard Padmore ${ }^{1}$, Jungjin Park ${ }^{1,5}$, Anthony Warwick ${ }^{1}$ and Young-Sang $\mathrm{Yu}^{1}$

${ }^{1 .}$ Advanced Light Source, Lawrence Berkeley National Lab, Berkeley, CA, 94720, USA

${ }^{2}$ Department of Physics, University of California at Berkeley, Berkeley, CA 94720, USA

${ }^{3}$ Engineering Division, Lawrence Berkeley National Lab, Berkeley, CA, 94720, USA

${ }^{4 .}$ Computational Research Division, Lawrence Berkeley National Lab, Berkeley, CA, 94720, USA

${ }^{5 .}$ School of Chemical and Biological Engineering, Seoul National University, Seoul, Republic of Korea.

* Corresponding author, dashapiro@1bl.gov

The Advanced Light Source (ALS) has recently commissioned a new beamline for coherent scattering and microscopy with soft x-rays. Called COSMIC, the beamline has two branches: one dedicated to coherent imaging with soft $\mathrm{x}$-rays in the energy range of 250 to $2500 \mathrm{eV}$ and the other dedicated to coherent scattering in the x-ray energy range 250 to $1700 \mathrm{eV}[1,2]$. The beamline optical design is intended to maximize coherent flux for brightness limited experiments, such as scanning microscopy, while also providing energy resolution suitable for near-edge x-ray absorption spectroscopy. Thus the scope of the beamline performance is well suited to the study of chemical, magnetic and morphological phases of nanomaterials.

The beamline design was previously described but is briefly mentioned here for completeness $[1,2]$. The first optic is a vertically collimating cylinder that absorbs about 300 Watts of power with the insertion device, an elliptically polarizing undulator with $38 \mathrm{~mm}$ period, set to the minimum gap of 10 $\mathrm{mm}$. A gap this small is only possible within the ALS storage ring with the use of non-evaporable getter (NEG) pumping within the straight section. The COSMIC straight section is the first NEG vacuum chamber in use at the ALS. The entrance-slitless monochromator is a collimated plane grating with either 100 or 300 lines per mm, each have two coating stripes: one gold and one rhodium. This monochromator design is flexible in that allows for either high flux or higher order suppression operational modes. In both branches, which are separated by a movable switching mirror, the monochromator illuminates a toroidal mirror that focuses onto an adjustable square exit slit. The Imaging branch exit slit illuminates a 240 micrometer diameter zone plate three meters downstream and within the endstation.

The COSMIC Imaging beamline is dedicated to coherent imaging using diffractive methods in order to surpass practical resolution limits imposed by conventional x-ray focusing optics [3-5]. The need for ptychographic imaging is exacerbated somewhat for longer wavelength x-rays because of the very short working distances required for high-resolution optics and because of the availability of high coherent flux and sample contrast which are needed to surpass conventional optical performance [6]. As an example, a target resolution of $5 \mathrm{~nm}$ at $1 \mathrm{keV}$ photon energy would require an optic with $1 \mathrm{~mm}$ focal length using standard geometries. This puts the order-sorting aperture (OSA) within 300 microns of the sample and inhibits the use of complex sample environments. Ptychographic imaging has been shown to improve the spatial resolution beyond the spot size by more than an order of magnitude. This is a 

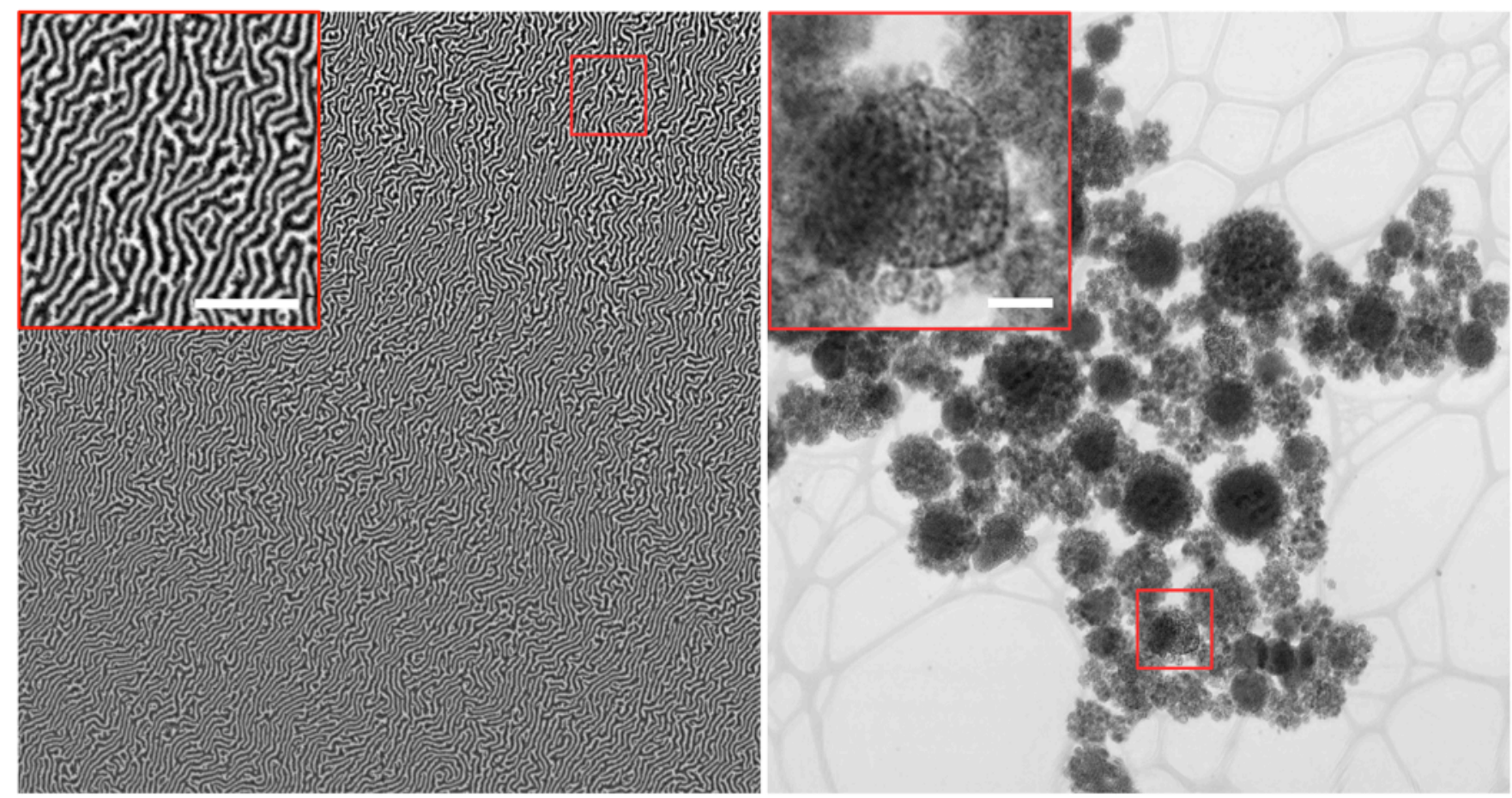

Figure 1 (Left) Conventional STXM image of a FeGd multilayer film using circularly polarized x-rays at the $\mathrm{Fe}_{3}$ absorption edge of $708 \mathrm{eV}$. The sample was illuminated by a $45 \mathrm{~nm}$ outer zone width optic and scanned on a $30 \mathrm{~nm}$ grid. Inset shows clear magnetic domains of about $100 \mathrm{~nm}$ width. Full field of view is 40 microns and scale bar is 1 micron. A linear transmission gradient was removed for clarity. (Right) Magnitude of a ptychographic reconstruction of a Fe containing compound used in battery anode applications. Secondary particles are up to a micron in size while primary particles are about $10 \mathrm{~nm}$ in size. The reconstructed pixel size is $5 \mathrm{~nm}$. Full field of view is 10 microns and scale bar is 200 nanometers.

practical limit related to both detector performance and sample contrast. Thus, a $45 \mathrm{~nm}$ focusing optic could be used to provide $9 \mathrm{~mm}$ focal length while also making few-nanometer resolution by ptychography achievable. The new scanning microscope at the COSMIC beamline is an imaging platform designed around such an optic and achieves rapid switching between conventional scanning microscopy with moderate spatial resolution and ptychographic imaging with high resolution. Switching is achieved automatically by motorized motion of the total transmission detector, a small area silicon PIN diode, out of the solid angle of the CCD during diffraction measurements.

The microscope is based upon a compact design with four key technologies: novel opto-mechanics for precise and fast scanning of the zone plate relative to the sample, co-scanning of the OSA with the zone plate, an ultra-stable sample stage (incorporating coarse, global positioners) compatible with commercial TEM sample holders and the use of a fast frame rate charge coupled device detector (CCD) [7]. At three meters from the beamline exit slit, the zone plate is overfilled and is uniformly illuminated across its full 100 micron, which is manipulated by a custom three-axis piezo stage. The two axes orthogonal to the beam direction (imaging plane) are flexure actuators while the motion along the beam direction (focusing) is a custom piezo stick-slip drive actuated by shear actuators against two flat surfaces of a sapphire rod with octagonal cross-section. Two other surfaces of this octagon are gold coated and serve as integrated mirrors for laser interferometry. A laser interferometer measures zone plate displacements relative to the metal vessel to which the sample stage is mounted. Vibrational stability at the zone plate 
is $0.5 \mathrm{~nm}$ RMS while sample drift measured relative to the optical axis is less than 2 nanometers per minute. The microscope sits on a passively damped support structure, with negligible transmission of vibrations above $13 \mathrm{~Hz}$, and within an environmental enclosure with active temperature control.

Commissioning experiments initially focused on the use of lower energy x-rays, in the range 500 to $1000 \mathrm{eV}$, where coherent flux is very high and the availability of the L-absorption edges for several transition metals leads to high sample contrast and use within a wide range of energy science applications. As a brief presentation of two advanced capabilities we present images of the magnetic domain pattern within a $90 \mathrm{~nm}$ thick FeGd film (Figure 1, left) and of a nano-structured variety of $\mathrm{Fe}_{3} \mathrm{O}_{4}$ used in battery anode applications (Figure 1, right). Using circularly polarized light and conventional scanning microscopy, the magnetic domain structure becomes apparent in the FeGd film while combinations of data from both polarization directions would allow for quantification of the magnetization. Representing one sixth of the total viewable area, this image shows the ability to visualize weak contrast nano-structure over a large field of view with a zone plate limited Raleigh resolution of $55 \mathrm{~nm}$. The contrast between the magnetic domains is $\sim 15 \%$ and indicates a high degree of circular polarization. Meanwhile, the ptychographic image demonstrates the ability to achieve diffraction limited resolution while imaging high contrast nano-structures. In this case, a resolution of $11 \mathrm{~nm}$ is achieved, as estimated by Fourier Ring Correlation with half-bit threshold, using $200 \mathrm{~ms}$ exposures. This is a conservative estimate as it requires independent reconstructions, each using half of the available diffraction data. The ptychographic data were recorded on a $40 \mathrm{~nm}$ scan grid with 62500 points and were analyzed using the SHARP software package [8]. A custom data pipeline, which moves diffraction data from the detector and through a pre-processing step in preparation for ptychographic analysis, is described separately.

The next commissioning phase will involve optimization of advanced sample environments, such as commercial fluid-flow electrochemistry TEM holders, and sample rotation for nano-tomography measurements. We are also collaborating with the ALS detector group on implementing a fully columnparallel CCD that will be 100X faster than the current generation CCD. CCD readout currently accounts for half of the experiment time.

[1] T. Warwick, et al, LSBL-1033RevA, 2012.

[2] D.A. Shapiro, et al, Journal of Physics: Conference Series, 425 (19), 2012.

[3] P. Thibault, et al, Science 321, 379-382 (2008).

[4] M. Dierolf, et al, Nature 467, 436-439 (2010).

[5] D.A. Shapiro, et al, Nat. Photonics 8, 765 (2014).

[6] W. Chao, et al, Nature, 435, 1210 (2005).

[7] P. Denes, et al, Rev. Sci. Instrum. 80, 083302 (2009).

[8] S. Marchesini, et al, Journal of Applied Crystallography 49 (4), (2016)

[9] The ALS is supported by the Director, Office of Science, Office of Basic Energy Sciences, of the US Department of Energy (contract no. DE-AC02-05CH11231). This work is partially supported by the Center for Applied Mathematics for Energy Research Applications (CAMERA), which is a partnership between Basic Energy Sciences (BES) and Advanced Scientific Computing Research (ASRC) at the US Department of Energy 
https://doi.org/10.1017/S1431927618012485 Published online by Cambridge University Press 